\title{
Retrieval of the total precipitable water vapor and cloud liquid water path over ocean from the Feng-Yun 3D microwave temperature and humidity sounders
}

\author{
Jun Yang ${ }^{1, *}$, Fuzhong Weng ${ }^{1}$, Hao $\mathrm{Hu}^{1}$, Peiming Dong ${ }^{1}$ \\ $5{ }^{1}$ State Key Laboratory of Severe Weather, Chinese Academy of Meteorological Sciences, Beijing 100081, \\ China \\ Correspondence to: Jun Yang (yangjun@cma.gov.cn)
}

\begin{abstract}
Feng-Yun 3D (FY-3D) satellite is the latest polar-orbiting meteorological satellite launched by China and carry 10 instruments onboard. Its microwave temperature sounder (MWTS) and microwave humidity sounder (MWHS) can acquire a total of 28 channels of brightness temperatures, providing rich information for profiling atmospheric temperature and moisture. However, due to a lack of two important frequencies at 23.8 and $31.4 \mathrm{GHz}$, it is difficult to retrieve the total precipitable water vapor (TPW) and cloud liquid water path (CLW) from FY-3D microwave sounder data as commonly done for other microwave sounding instruments. Using the channel similarity between Suomi NPP advanced technology microwave sounder (ATMS) and FY3D microwave temperature and humidity sounders, a machine learning technique is used to generate the two missing low frequency channels of MWTS and MWHS. Then, a new data set named as a combined microwave sounder (CMWS) is obtained and has the same channel setting as ATMS but the spatial resolution is consistent with MWTS. It is shown that the mean absolute errors of the two simulated channels are both between 3 and 4 $\mathrm{K}$. The simulation errors mainly distribute in the high latitude regions, coastlines and the boundaries of some heavy rainfall. A statistical inversion method is adopted to retrieve TPW and CLW over oceans from the FY-
\end{abstract} 3D CMWS. The inter-comparison between different satellites shows that the inversion products of FY-3D CMWS and Suomi NPP ATMS have good consistency in magnitude and distribution.

\section{Introduction}

Clouds and water vapor are vital for regulating the global radiation budget, and their distribution and evolution 
(RAOB) can obtain direct measurement of water vapor, but the operational RAOB measurements are only performed twice a day at several hundred fixed stations around the world, which makes its temporal and spatial resolution have great limitations (Gui et al., 2017). Therefore, remote sensing inversion has become an important means to obtain TPW and CLW. Both ground-based and satellite retrieval methods have been widely developed in the microwave range to get local or global cloud water content. Ground-based microwave radiometer can obtain continuous TPW and CLW measurements with a very high temporal resolution for a stationary observation site (Lin et al., 2001; Cadeddu et al., 2009; Cimini et al., 2010; Yang and Min, 2018). The limitation of ground observation lies in its poor spatial distribution, especially on the ocean.

Compared with ground-based observation, satellite measurement can provide TPW and CLW inversion products on a global scale although its temporal resolution is relatively low. The large-scale TPW and CLW distribution obtained by satellite inversion, in addition to being used for global cloud water resource assessment, another important function is to use as cloud detection criterion for quality control in satellite data assimilation. The brightness temperature measured by satellites is affected by many factors such as surface emissivity, water vapor and cloud liquid water content. In the ocean, the surface emissivity is relatively uniform. Normally, TPW and CLW can be retrieved using two microwave channel measurements. In the early days of satellite applications, Grody et al. (1980) applied the data from 21 and $31 \mathrm{GHz}$ channels of scanning microwave spectrometer mounted on Nimbus 6 to retrieve CLW over the Pacific Ocean. Prabhakara et al. (1983) developed a method to retrieve CLW from the data on Nimbus 7 scanning multichannel microwave radiometer at 6.6 and 10.7 GHz. With the launch of special sensor microwave/imager (SSM/I) aboard the defense meteorological satellite program, a large number of satellite inversion algorithms based on this passive microwave sensor were reported to obtain globe distribution of TPW and CLW over ocean (Greenwald et al., 1993; Liu and Curry, 1993; Weng and Grody, 1994; Ferraro et al., 1996). There are four frequencies (at 19.35, 22.235, 37 and 85.5 $\mathrm{GHz}$ ) in SSM/I, and the existing algorithms mainly use the combination of $22.235 \mathrm{GHz}$ and another frequency to invert TPW and CLW. The successive launch of advanced microwave sounding unit and advanced technology microwave sounder (ATMS) further promoted the application of satellite inversion of TPW and CLW over ocean (Dyras and Serafin-Rek, 2002; Mo and Liu, 2008; Weng et al., 2012). Both statistical (Grody et al., 2001) and physical inversion (Weng et al., 2003) methods had been proposed to improve the inversion accuracy of TPW and CLW using two window channels (at 23.8 and $31.4 \mathrm{GHz}$, respectively). 
Feng-Yun 3D (FY-3D) satellite, which was launched in November 2017, is the fourth satellite of China's second-generation polar-orbiting satellite. There are 10 sets of advanced remote sensing instruments on the FY3D, among which microwave temperature sounder (MWTS) and microwave humidity sounder (MWHS) are mainly designed to obtain atmospheric temperature and water vapor profiles. The MWTS operates in an oxygen absorption band of 50-60 GHz and is subdivided into 13 channels. There are total 15 channels in MWHS, which are located in oxygen absorption zone ( 8 channels near $118.75 \mathrm{GHz}$ ), water vapor absorption zone ( 5 channels near $183.31 \mathrm{GHz}$ ) and window zone (at 89 and $150 \mathrm{GHz}$ ), respectively. Using the dual oxygen absorption bands in MWTS and MWHS, several pairs of oxygen channels can be applied to compute cloud emission and scattering index at different height levels (Han et al., 2015), where each pair of oxygen channels has a similar peak weighting function height. The strong water vapor absorption channels at high frequency in MWHS are very sensitive to small changes of water vapor, and it is only suitable for inversion of TPW below $7 \mathrm{~kg} / \mathrm{m}^{2}$ (Melsheimer and Heygster, 2008). Therefore, it is often used for water vapor retrieval in polar regions. Two window channels in MWHS can be used to retrieve cloud scattering index (Bennartz et al., 2002) and cloud ice water path (Zhao and Weng, 2002). Although some cloud water information can be obtained using different algorithms based on the existing microwave channels of FY-3D, it is still very difficult to give specific values of TPW and CLW. The main reason is the lack of two important window channels, 23.8 and $31.4 \mathrm{GHz}$, in FY3D. Fortunately, compared with ATMS aboard Suomi NPP satellite, MWTS and MWHS contain all ATMS channel information except two low-frequency window channel and thus, ATMS data provide a good opportunity for simulating FY-3D MWTS measurements at any frequencies. two window channels, which makes it possible to retrieve TPW and CLW from FY-3D microwave sensors. In the following section, the channel simulation method based on ML is first discussed. Some ML simulation results and accuracy assessments are presented in section 3. In section 4, the inversion results of TPW and CLW based on FY-3D are introduced and compared with those of ATMS. The brief conclusions and recommendations for future work are discussed in section 5 .

\section{Channel simulation algorithm based on machine learning}

For any field of view (FOV), the measurements of each channel on the same satellite sensor should have a certain correlation, because their corresponding surface types and atmospheric environment are exactly the 
same. Therefore, we can establish the relationship between two low-frequency window channels and other channels of ATMS through the ML model for all FOVs. Since MWTS and MWHS contain all channel settings in ATMS except for the two low-frequency window channels, we can match FY-3D data to ATMS level by cross calibration, thus realizing the prediction of missing channel values in FY-3D using ATMS training model. The flow chart of the proposed channel simulation algorithm is shown in Figure 1, which includes three main steps: footprint matching, cross calibration and machine learning. Firstly, each channel of MWHS transforms to a new channel consistent with the spatial resolution of MWTS by performing footprint matching with MWTS. Then we can get a 28-channel data set, 20 of which have the same channel properties as ATMS. Secondly, these 20 channels will be cross-calibrated with the corresponding channels in ATMS. Thirdly, the typical ATMS samples are trained using ML method, and the model relationship between other channels and two lowfrequency window channels in ATMS can be established. Next, the corresponding 20 channels in FY-3D are input into the model to obtain two simulated low-frequency channels. It will combine 20 channels in FY-3D to form a new data set, named combined microwave sounder (CMWS), which is identical to the channel settings of the ATMS, but has the same observation range and resolution as the MWTS. Several key steps will be introduced in the following subsections.

\subsection{Footprint matching}

Although both MWTS and MWHS are mounted on the FY-3D satellite, they are two completely independent sensors, which also results in the difference of FOV size and FOV number on each scan line between MWTS and MWHS. For example, there are 98 FOVs on each scan line in MWHS, while only 90 FOVs in MWTS. In order to make the pixels corresponding to MWTS and MWHS have the same observation position and instantaneous FOV, we need to perform footprint matching for the original observation data. Since the spatial resolution of MWHS is higher than that of MWTS, an alternative method is to use B-G method (Backus and Gilbert, 1968) to re-sample MWHS to produce observations with the same resolution as MWTS. However, on the FY-3D satellite, the scan times of MWTS and MWHS does not match well, which makes the brightness temperatures observed by them do not overlap well in space. Therefore, we adopted a simple weighted average to match the MWHS observations to the MWTS resolution level. By setting a distance threshold, for each FOV in the MWTS, find all points below the threshold in the MWHS, and calculate the average of these points to obtain the matched MWHS brightness temperature values. Figure 2 shows two typical MWHS channels ( 89 and $183.31 \pm 1.0 \mathrm{GHz}$ ) on July 9, 2018 and their results after footprint matching with MWTS. The left column 
represents the original measurements of MWHS, and the right column is the results after matched. The matched results are very close to the original observations in both intensity and distribution of brightness temperature, which ensures that our footprint matching does not lose too much information.

\subsection{Cross calibration}

Although the channel settings between ATMS and CMWS are basically the same, the brightness temperatures of ATMS and CMWS come from different satellite measurements, which inevitably leads to a certain deviation between the two observations due to the differences in hardware processing and radiometric calibration. Since our training samples are all from ATMS, and the input data of the model prediction comes from CMWS, it becomes indispensable to perform a cross calibration process, which will ensure the measured values on each channel between the two instruments are as consistent as possible.

The sub-satellite trajectories of Suomi NPP and FY-3D satellites are very close each other on February 1-2, 2018, which allows us to use the data of these two days to cross-calibrate ATMS and CMWS. For each FOV in CMWS, the arc length and observation time difference between the pixel and all observation points in ATMS are calculated. The pair of FOV with the shortest distance may be the observation of the same point by two sensors. By setting a distance threshold and a time threshold, all matching pairs of FOVs satisfying the threshold conditions can be considered as the same observation point and used for cross calibration. A simple linear regression method is adopted to derive a linear regression equation for each channel, which can match the observations of CMWS to the level of ATMS. Table 1 provides the linear fitting coefficients (slope and intercept) and mean absolute error for each channel.

\subsection{Machine learning}

After completing the footprint matching of MWTS and MWHS and the cross calibration between ATMS and CMWS, the next key step of channel simulation is the application of ML algorithm. ML can build an effective training model by learning finite samples, which can not only predict the known data but also the unknown data. ML can be divided into supervised learning and unsupervised learning. Under supervised learning, each group of training data has a clear mark or result. In unsupervised learning, data is not specifically identified, and learning model is designed to infer some of the intrinsic structure of data. The issue to be solved in this paper is a regression problem, so supervised learning should be adopted to train the model. Traditional supervised 
machine, decision tree, etc. Recently, the ensemble algorithms have been developed, which solve the single prediction problem by establishing a combination of several models (Rokach, 2010). It works by generating multiple classifiers/models, each of which learns and makes predictions independently. These predictions are ultimately combined into a single prediction, which is usually better than any single estimator. 2001). Its basic unit is the decision tree, and each tree is bootstrapping random sampling from the training set. There is no correlation between each decision tree in a RF model. The basic schematic diagram of the RF is shown in Figure 3. A decision tree consists of a root node, internal nodes and leaf nodes. First, the root node splits according to randomly selected data set attributes, and then similar splitting process is repeated in the internal nodes until the node satisfies the maximum depth or reaches the maximum number of leaf nodes. Each leaf node returns a predicted value. The predicted result of a decision tree can be acquired by averaging the predicted values of all leaf nodes on the tree. Finally, the results of each decision tree are averaged to improve the ultimate predictive accuracy and control over-fitting. RF has the following main advantages: 1) Training can be highly parallelized, which makes training speed of the model have great advantages, especially for large samples such as satellite data; 2) Because the decision tree node splitting feature can be selected randomly, the training model can still be efficient even if the dimension of sample features is very high; 3) The training model has small variance and strong generalization ability because of random sampling.

\section{Accuracy evaluation and channel simulation examples}

In ATMS, there are remaining 20 channels besides two low-frequency window channels. To determine whether all 20 channels should be involved in the training of the RF model, we conducted some sensitivity tests based on a very popular ML software package scikit-learn (https://scikit-learn.org/stable/index.html). In order to facilitate the pixel-by-pixel quantitative evaluation of the simulation results, the data in the training set and the test set are all from ATMS. The full-day data of February 1, 2018 was chosen as the training set, and the data of February 2, 2018 was adopted as the test sample. By comparing the simulation results with the actual observations, the mean absolute error (MAE) of the two low-frequency window channels (represented by Ch1 and $\mathrm{Ch} 2$ respectively) in different channel combinations and different model parameters can be obtained. The simulated error of each single channel is displayed in the left panel of Figure 4. It is clear that MAEs of channels $3,4,5$ and 16 are significantly lower than those of other channels. Considering that the weighting functions and 
peak heights of these four channels are very similar to those of $\mathrm{Ch} 1$ and $\mathrm{Ch} 2$ (see the Figure 2 in Weng et al., 2012), it has natural advantages to simulate $\mathrm{Ch} 1$ and $\mathrm{Ch} 2$ using these four channels. In addition to these four channels, other channels in ATMS are added to the training model one by one to test their effects on the simulation accuracy of Ch1 and Ch2. The right panel of Figure 4 denotes different MAEs when each channel is progressively added to the training model. The trend of simulation accuracy for $\mathrm{Ch} 1$ and $\mathrm{Ch} 2$ is basically the same. When the channel used in the model increases from channel 6 to channel 11, the MAE is decreasing, and from channel 12 to channel 15, the MAE is starting to increase again. Channel 17 to channel 22 are high frequency channels in ATMS. When channel 17 and channel 18 are added to the training model, the simulation errors of $\mathrm{Ch} 1$ and $\mathrm{Ch} 2$ can be further reduced, but the introduction of channel 19 to channel 22 makes the MAEs of $\mathrm{Ch} 1$ and $\mathrm{Ch} 2$ slightly increased again. These sensitivity tests show that channel 12 to channel 15 and channel 19 to channel 22 cannot play a positive role in the simulation of $\mathrm{Ch} 1$ and $\mathrm{Ch} 2$. Therefore, in the next analysis, we totally adopted 12 channels to simulate the missing channels at frequencies 23.8 and $31.4 \mathrm{GHz}$ in FY-3D. In a RF model, in order to obtain the highest prediction accuracy and reduce the computation time, it is necessary to optimize some input parameters. We did some sensitivity tests to determine the values of these parameters (see Figure 5). The more decision trees are used in the RF model, the higher the accuracy of the model is, but the number of trees seriously affects the training speed. It is a reasonable choice to adopt 130 decision trees in our RF model. The maximum depth of each tree determines the extent of tree expansion. More depth does not significantly increase the accuracy of the model, so we set the maximum depth to 30 . The number of features to consider when looking for the best split obviously affects the accuracy of the model, and the simulation error is the smallest when all 12 channels are used. The other parameters, such as the maximum number of leaf nodes, the minimum number of samples required to split an internal node, the minimum number of samples required to be at a leaf node, can obtain the best simulation accuracy by directly using the default values of the software package scikit-learn.

Using the training channels and ML parameters determined by the aforementioned sensitivity tests, the two ATMS low frequency window channels on July 10, 2018 were simulated. In order to increase the representativeness of training samples, our training set consists of two categories; one is to select one-day data from each month in the past year, which mainly represents the basic global climate trends in different seasons. The other type is the data of the day before the forecast sample, which mainly on behalf of the global approaching weather system. The point-to-point accuracy evaluation results for Chl simulation are shown in 
Figure 6. The top row of Figure 6 represents the original ATMS measurement and ML simulated result for Ch1 on July 10, 2018. The bottom row of Figure 6 shows the difference between observation and simulation, as well as the scatter density of observed and simulated brightness temperatures. The similar accuracy evaluation images for $\mathrm{Ch} 2$ simulation are depicted in Figure 7. Visually, the range of error distribution of $\mathrm{Ch} 1$ and $\mathrm{Ch} 2$ simulation is very similar, which mainly distributed in the high latitude regions, coastlines and the edge of some heavy rainfall. It should be noted that the MAE of Ch1 simulation is slightly bigger than that of $\mathrm{Ch} 2$ simulation, corresponding to $3.5252 \mathrm{~K}$ and $3.4151 \mathrm{~K}$ respectively. previously. We can get $\mathrm{Ch} 1$ and $\mathrm{Ch} 2$ simulation results based on FY-3D observation data by replacing the predictive input of ML model with FY-3D measurements. Figure 8 shows the simulation results of the two lowfrequency window channels based on FY-3D observations on September 13, 2018. The left column is the actual observations of ATMS, and the right column is the simulation results based on FY-3D observations. The upper row is the observation and simulation of $23.8 \mathrm{GHz}$ and the lower row is the related results of $31.4 \mathrm{GHz}$. It can be seen that the simulation results of the two channels are very similar to the observations of the ATMS, especially on the ocean. On September 13, 2018, the super typhoon "Mangkhut" (about at $15^{\circ} \mathrm{N}, 130^{\circ} \mathrm{E}$ ) is developing vigorously in the Northwest Pacific Ocean. It is clear that our simulation results for both Ch1 and Ch2 can give the accurate locations and basic form of the typhoon. It should be pointed out that the results of the simulation using FY-3D will definitely be lower than the accuracy of quantitative evaluation by ATMS. This is because the cross calibration between ATMS and FY-3D will inevitably introduce some new errors. Typically, the simulation error of the north and south poles is relatively large, and the simulated brightness temperature of the typhoon area is still a little weak.

\section{Retrieval of the total precipitable water vapor and cloud liquid water path}

Traditionally, retrieving TPW and CLW from satellite measurements is mainly aimed at the ocean surface because of its relatively low and uniform surface emissivity. Although several methods have been reported to retrieve TPW from land surface (Aires et al., 2001; Boukabara et al., 2010; Zhou et al., 2016), it is still very difficult to retrieve TPW and CLW from non-oceanic surface because of its pixel-by-pixel high uncertainty of surface emissivity. The purpose of this paper is not to propose a new inversion method, mainly to test the 
of TPW and CLW mainly focuses on the ocean. After the high-precision simulation of 23.8 and $31.4 \mathrm{GHz}$ channels based on FY-3D measurements, the inversion of TPW and CLW over ocean becomes relatively easy. There are two inversion methods over ocean widely used in the field of microwave remote sensing, one is Grody's statistical inversion method (Grody et al., 2001) and the other is Weng's physical inversion method (Weng et al., 2003). In theory, physical inversion method should have higher retrieval accuracy than statistical inversion method. However, in Weng's physical inversion model (Weng et al., 2003), some precise ocean and cloud parameters need to be input, such as sea surface temperature, wind speed and cloud layer temperature. These parameters are usually difficult to obtain precise measurements. An alternative is to get them from global forecast system or from reanalysis data. Considering the RMS error for non-precipitation CLW retrieval is no more than $0.05 \mathrm{~mm}$ (Grody et al., 2001), we select Grody's statistical method to retrieve TPW and CLW over ocean from FY-3D in this paper. The equations for statistical inversion can be expressed as,

$\mathrm{TPW}=\cos \theta\left[a_{0}+a_{1} \ln \left(T_{s}-T_{b 23}\right)+a_{2} \ln \left(T_{s}-T_{b 31}\right)\right]$

$\operatorname{CLW}=\cos \theta\left[b_{0}+b_{1} \ln \left(T_{s}-T_{b 23}\right)+b_{2} \ln \left(T_{s}-T_{b 31}\right)\right]$

where $\theta$ is the local zenith angle, $T_{S}$ is the surface temperature, $T_{b 23}$ and $T_{b 31}$ represent the observed brightness temperatures of 23.8 and $31.4 \mathrm{GHz}$, respectively. To ensure $T_{s}$ is larger than $T_{b 23}$ and $T_{b 31}$ over ocean, the value of $T_{s}$ is set to $285 \mathrm{~K}$. The coefficients $a_{0}, a_{1}, a_{2}, b_{0}, b_{1}$, and $b_{2}$ can be obtained by performing regression analysis on the simulated channel measurements. The specific coefficients are as follows,

$a_{0}=247.92-(69.235-44.177 \cos \theta) \cos \theta$

$a_{1}=-116.27, a_{2}=73.409$

$b_{0}=8.240-(2.622-1.846 \cos \theta) \cos \theta$

$b_{1}=0.754, b_{2}=-2.265$

Using the above formulas and combining the brightness temperature values of 23.8 and $31.4 \mathrm{GHz}$ channels simulated in CMWS, the specific values of TPW and CLW on the ocean can be obtained efficiently. The validation of TPW retrieved from satellite can be carried out by various methods. On the one hand, it can be compared with the direct measurements of RAOB or the inversion results of ground-based microwave radiometer; on the other hand, it can also be done by inter-comparing with the inversion product of other satellites. The accuracy verification of the CLW is mainly compared with the retrieval results of the inversion 
of ground-based microwave radiometer or the retrieval product of other satellites. Here, we mainly verify the inversion effect of TPW and CLW by comparing with ATMS inversion results. Figure 9 and Figure 10 show the inversion results on July 8, 2018 and September 11, 2018, respectively. Each of these two days has a super typhoon developing in the Northwest Pacific Ocean, corresponding to the typhoon "Maria" and "Mangkhut". To better increase the diversity of data, Figure 9 shows the inversion results of the descending orbit, and Figure 10 denotes the results of the ascending orbit. From the comparison of two examples, the inversion results of TPW and CLW based on FY-3D CMWS are very similar to those of ATMS inversion both in intensity and distribution.

\section{Conclusion and discussion}

FY-3D satellite is the latest polar-orbiting meteorological satellite launched by China, which carries 10 sets of advanced monitor instruments. The MWTS and MWHS mounted on the FY-3D provide a total of 28 channel observations that greatly improve the inversion accuracy of temperature and humidity profiles. In particular, the 50-60 GHz oxygen channels in the MWTS and the oxygen channels near the $118.75 \mathrm{GHz}$ in the MWHS can be used not only for mutual backup but also for retrieving of clouds and precipitation using dual oxygen absorption channels. However, due to the lack of two important low-frequency window channels, at frequencies 23.8 and $31.4 \mathrm{GHz}$, it is hard to retrieve TPW and CLW using FY-3D microwave measurements. The similarity of channel settings between NPP ATMS and FY-3D MWTS\&MWHS provides a good opportunity for us to simulate these two missing channels. Firstly, based on the correlation between measurements of all channels on the same sensor, the model relationship between other channels and two low-frequency window channels in ATMS is established by ML algorithm. Then cross calibration between MWTS and MWHS measurements and corresponding ATMS channels is carried out. Finally, the calibrated FY-3D measurements are input into the training model to obtain the simulated values. The results of quantitative evaluation show that the MAEs of the two channels are both between 3 and $4 \mathrm{~K}$, and the maximum error mainly occurs in high latitude regions, coastlines and the vicinity of some heavy rainfall. Especially in the central region of typhoon, the simulated brightness temperatures are somewhat lower than those ATMS measurements. It should be pointed out that although the simulation errors of the two missing channels still seem to be a little large, the simulation accuracy is sufficient to meet the quality control requirements of satellite data assimilation and atmospheric profile 
parameter inversion. In the next step, we will build training models for ocean and land respectively, so as to further improve the accuracy of channel simulation.

After simulated the 23.8 and $31.4 \mathrm{GHz}$ channels using FY-3D microwave observations, Grody's statistical method was adopted to reverse TPW and CLW over ocean. By comparing with different satellite inversion products, it can be found that the inversion results of TPW and CLW based on FY-3D are in good agreement with those of ATMS in both strength and distribution. Actually, the channel settings of CMWS data set established by machine learning are basically consistent with ATMS, which makes the existing inversion algorithms based on ATMS can be seamlessly transplanted into CMWS. In addition, after the successful simulation of 23.8 and $31.4 \mathrm{GHz}$ channels, we can even compose a new CMWS data set of 30 channels (plus 13 channels of MWTS and 15 channels of MWHS), which will provide more details for the inversion of temperature and humidity profiles in the vertical direction. Thus, the new CMWS data set has great application prospects, especially in the inversion of typhoon warm core structure, typhoon location and intensity determination, and precipitation estimation. At the same time, the next FY-3 satellite FY-3E will soon be launched, in which two window channels (at 23.8 and $31.4 \mathrm{GHz}$ ) will be added in the new microwave sounder. The simulation method proposed in this paper can also provide a good proxy simulation for FY-3E.

Code and Data availability. Data used in this study can be made available upon request to the author.

Author Contributions. JY performed the calculus, designed and conducted the experiments and wrote the paper; FZW edited and supervised the form of the paper; HH validated the results; PMD provided help for coding design and modified the paper.

Competing interests. The authors declare that they have no conflict of interest.

Acknowledgements. This work is partly supported by the National Key Research and Development Program of China (2018YFC1506500) and the National Natural Science Foundation of China (41675030). We also gratefully acknowledge the support from National Satellite Meteorological Centre under agreement FY3 (02P)MAS-1803. 


\section{Reference}

Stephens, G. L.: Cloud feedbacks in the climate system: A critical review, J. Climate, 18, 237-273, https://doi.org/10.1175/JCLI-3243.1, 2005.

Yang, J., Min, Q., Lu, W., Ma, Y., Yao, W., and Lu, T.: An RGB channel operation for removal of the difference of atmospheric scattering and its application on total-sky cloud detection, Atmospheric Measurement Techniques, 10(3), 1191-1201. https://doi.org/10.5194/amt-10-1191-2017, 2017. Characteristics in the Shigatse Area of the Tibetan Plateau Using the Total-Sky Images, Journal of Applied Meteorology and Climatology, 57, 1977-1987. https://doi.org/10.1175/JAMC-D-18-0095.1, 2018.

Gui, K., Che, H., Chen, Q., Zeng, Z., Liu, H., Wang, Y., Zheng, Y., Sun, T., Liao, T., Wang, H., and Zhang, X.: Evaluation of radiosonde, MODIS-NIR-Clear, and AERONET precipitable water vapor using IGS groundbased GPS measurements over China, Atmos. Res., 197, 461-473, 2017.

Lin, B., Minnis, P., Fan, A., Curry, J. A., and Gerber, H.: Comparison of cloud liquid water paths derived from in situ and microwave data taken during the SHEBA/FIREACE, Geophysical Research Letters, 28(6), 975-978, https://doi.org/10.1029/2000GL012386, 2001.

Cadeddu, M. P., Turner, D. D., and Liljegren, J. C.: A neural network for real-time retrievals of PWV and LWP from arctic millimeter-wave ground-based observations, IEEE T. Geosci. Remote S., 47, 1887-1900, 2009.

Cimini, D., Westwater, E. R., and Gasiewski, A. J.: Temperature and humidity profiling in the Arctic using millimeter-wave radiometry and 1DVAR, IEEE T. Geosci. Remote S., 48(3), 1381-1388, https://doi.org/10.1109/TGRS.2009.2030500, 2010.

Yang, J., and Min, Q.: Retrieval of atmospheric profiles in the New York State Mesonet using one-dimensional variational algorithm, J. Geophys. Res.: Atmospheres, 123, 7563-7575, https://doi.org/10.1029/2018JD028272, 2018.

Grody, N. C., Gruber, A., and Shen, W. C.: Atmospheric water content over tropical pacific derived from the Nimbus-6 scanning microwave spectrometer, J. Appl. Meteorol., 8, 986-996, 1980.

Prabhakara, C., Wang, J., Chang, A. T. C., and Gloersen, P.: A statistical examination of Nimbus-7 SMMR data 
Greenwald, T., Stephens, G., Vonder Haar, T., and Jackson, D.: A physical retrieval of cloud liquid water over the global oceans using Special Sensor Microwave/Imager (SSM/I) observations, J. Geophys. Res., 98, 18,47118,488, 1993.

Liu, G., and Curry, J. A.: Determination of characteristic features of cloud liquid water from satellite microwave measurements, J. Geophys. Res., 98, 5069-5092, 1993.

Weng, F., and Grody, N. C.: Retrieval of cloud liquid water using the Special Sensor Microwave Imager (SSM/I), J. Geophys. Res., 99, 25,535-25,551, 1994.

Ferraro, R. R., Weng, F., Grody, N., and Basist, A.: An eight year (1987-1994) time series of rainfall, clouds, water vapor, snow and sea ice derived from SSM/I measurements, Bull. Am. Meteorol. Soc., 77, 891-905, 1996. Dyras, I., and Serafin-Rek, D.: The use of AMSU data from NOAA-15 satellite for meteorological products generation, Advances in Space Research, 30(11), 2461-2466, 2002.

Mo, T., and Liu, Q.: A study of AMSU-A measurement of brightness temperatures over the ocean, J. Geophys. Res., 113, D17120, doi:10.1029/2008JD009784, 2008.

Weng, F., Zou, X., Wang, X., Yang, S., and Goldberg, M. D.: Introduction to Suomi national polar-orbiting partnership advanced technology microwave sounder for numerical weather predict ion and tropical cyclone applications, J. Geophys. Res., 17, D19112, doi:10.1029/2012JD018144, 2012.

Grody, N., Zhao, J., Ferraro, R., Weng, F., and Boers, R.: Determination of precipitable water and cloud liquid water over oceans from the NOAA 15 advanced microwave sounding unit, J. Geophys. Res., 106, 2943-2953, 2001.

Weng, F., Zhao, L., Ferraro, R., Poe, G., Li, X., and Grody, N.: Advanced microwave sounding unit cloud and precipitation algorithms, Radio Sci., 38(4), 8068, doi:10.1029/2002RS002679, 2003.

Han, Y., Zou, X., and Weng, F.: Cloud and precipitation features of super typhoon Neoguri revealed from dual oxygen absorption band sounding instruments on board FengYun-3C satellite, Geophys. Res. Lett., 42, 916924, doi:10.1002/2014GL062753, 2015.

Melsheimer, C., and Heygster, G.: Improved retrieval of total water vapor over polar regions from AMSU-B microwave radiometer data, IEEE T. Geosci. Remote S., 46(8), 2307-2322, 2008.

Bennartz, R., Thoss, A., Dybbroe, A., and Michelson, D. B.: Precipitation analysis using the Advanced Microwave Sounding Unit in support of nowcasting applications, Meteorol. Appl., 9, 177-189, 2002. 
Zhao, L., and Weng, F.: Retrieval of ice cloud parameters using the advanced microwave sounding unit (AMSU), J. Appl. Meteorol. 41, 384-395, 2002.

Backus, G. E., and Gilbert, F.: The resolving power of gross earth data, Geophysical Journal of the Royal Astronomical Society, 16, 169-205, 1968.

Russell, S. J., and Norvig, P.: Artificial intelligence: a modern approach, Third Edition, Prentice Hall, 2010.

Rokach, L.: Ensemble-based classifiers. Artificial Intelligence Review, 33 (1-2), 1-39. http://dx.doi.org/10.1007/s10462-009-9124-7, 2010.

Breiman, L.: Random forests, Machine Learning, 45(1), 5-32, 2001.

Aires, F., Prigent, C., Rossow, W. B., and Rothstein, M.: A new neural network approach including first-guess for retrieval of atmospheric water vapor, cloud liquid water path, surface temperature and emissivities over land from satellite microwave observations, J. Geophys. Res., 106(D14), 14887-14907, 2001.

Boukabara, S. A., Garrett, K., and Wanchun, C.: Global coverage of total precipitable water using a microwave variational algorithm, IEEE T. Geosci. Remote S., 48(10), 3608-3621, 2010.

Zhou, F., Song, X., Leng, P., Wu, H.; and Tang, B.: An algorithm for retrieving precipitable water vapor over land based on passive microwave satellite data, Advances in Meteorology, 1-11. 
https://doi.org/10.5194/amt-2019-447

Preprint. Discussion started: 2 December 2019

(C) Author(s) 2019. CC BY 4.0 License.

\section{Figure Captions}

Figure 1. Flow chart of channel simulation algorithm based on machine learning.

Figure 2. Footprint matching examples for two typical channels.

Figure 3. Schematic diagram of random forest.

Figure 4. Simulation errors in different channel combinations.

Figure 5. Simulation errors under different RF model parameters.

Figure 6. Accuracy evaluation for Ch1 simulation.

Figure 7. Accuracy evaluation for $\mathrm{Ch} 2$ simulation.

Figure 8. Compared between the simulation and observation.

Figure 9. Comparison of retrieved TPW (top row) and CLW (bottom row) between ATMS (left column) and CMWS (right column) for the descending orbit measurement on July 8, 2018.

Figure 10. Comparison of retrieved TPW (top row) and CLW (bottom row) between ATMS (left column) and CMWS (right column) for the ascending orbit measurement on September 11, 2018. values) brightness temperatures. 
https://doi.org/10.5194/amt-2019-447

Atmospheric

Preprint. Discussion started: 2 December 2019

(c) Author(s) 2019. CC BY 4.0 License.

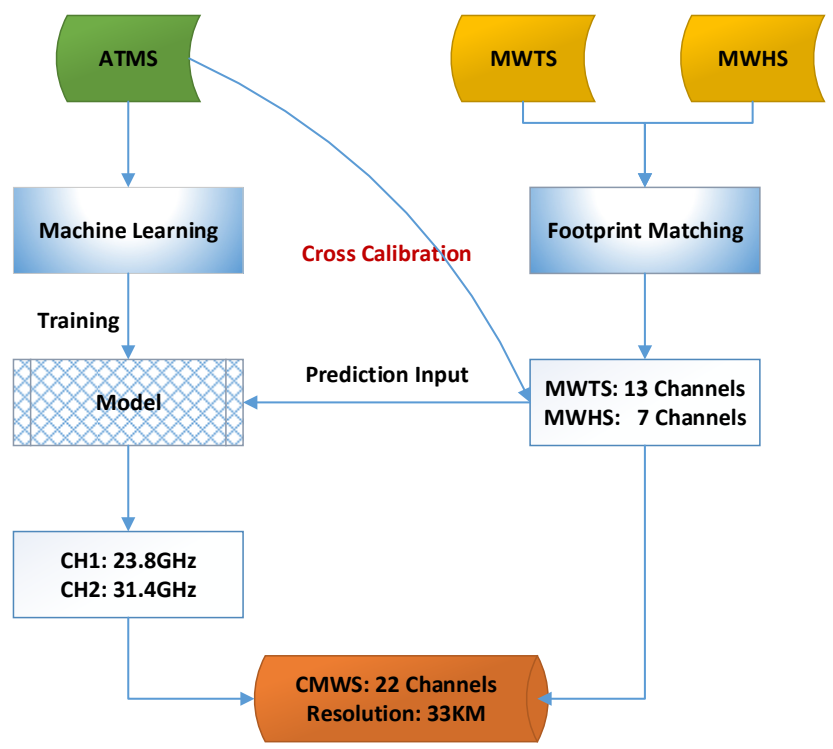

Figure 1. Flow chart of channel simulation algorithm based on machine learning. 
https://doi.org/10.5194/amt-2019-447

Preprint. Discussion started: 2 December 2019

(c) Author(s) 2019. CC BY 4.0 License.

\section{Atmospheric Measurement Techniques Discussions}
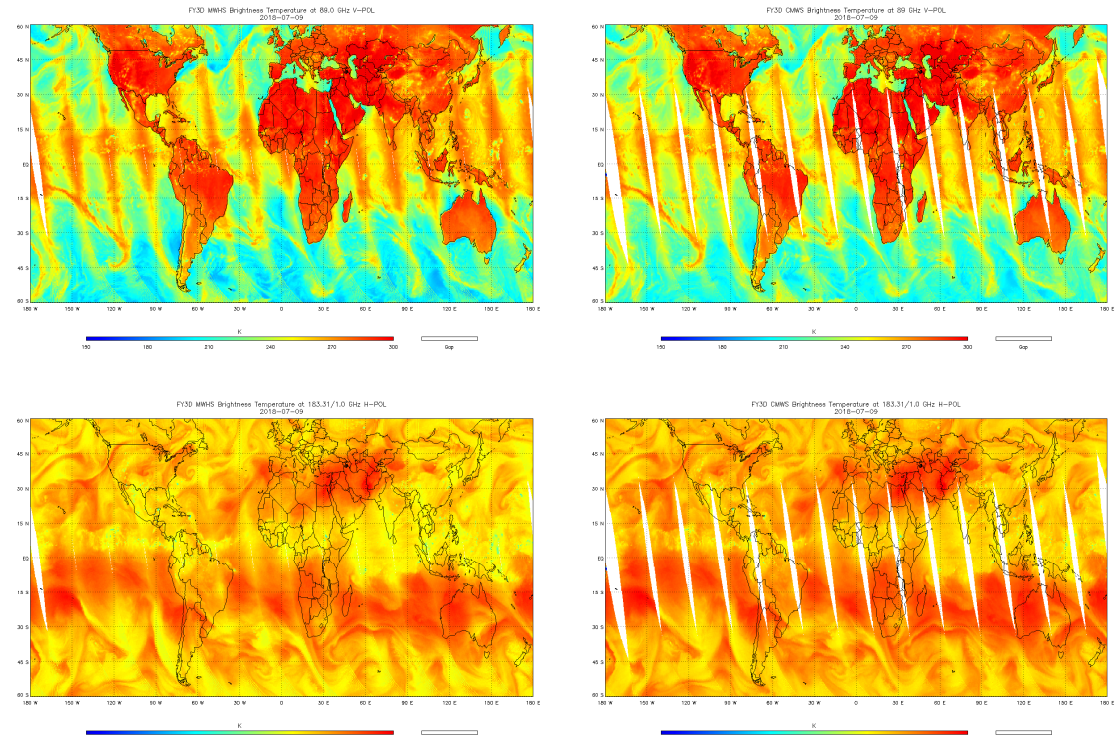

Figure 2. Footprint matching examples for two typical channels. (left column) the original measurements of MWHS, (right column) the resampled MWHS channels which have the same spatial resolution as MWTS channels. 


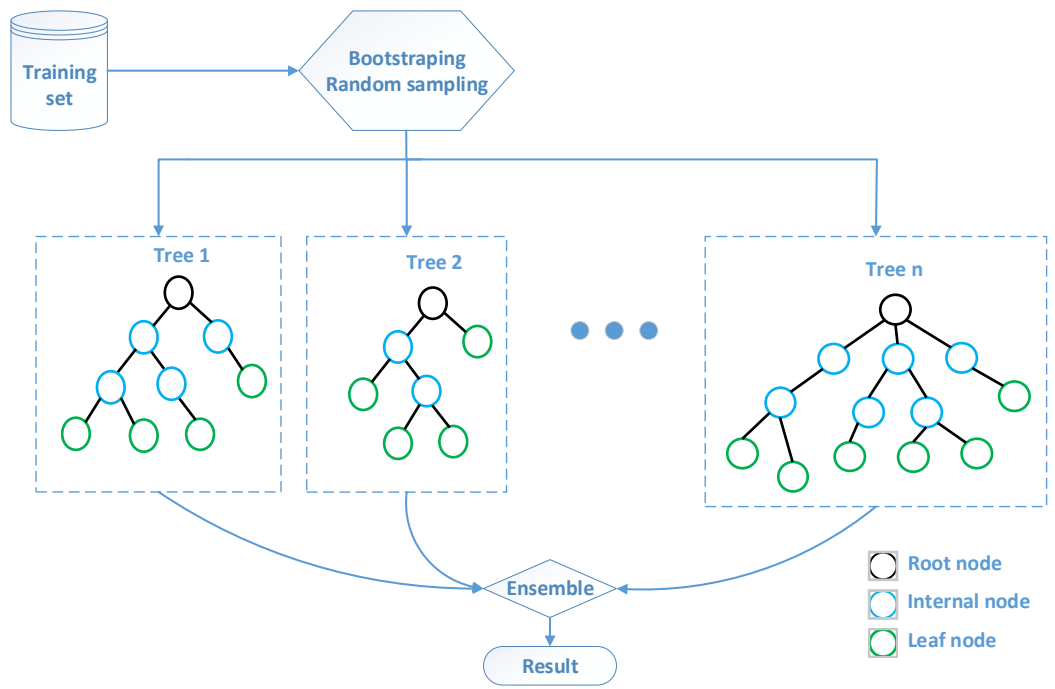

Figure 3. Schematic diagram of random forest. 

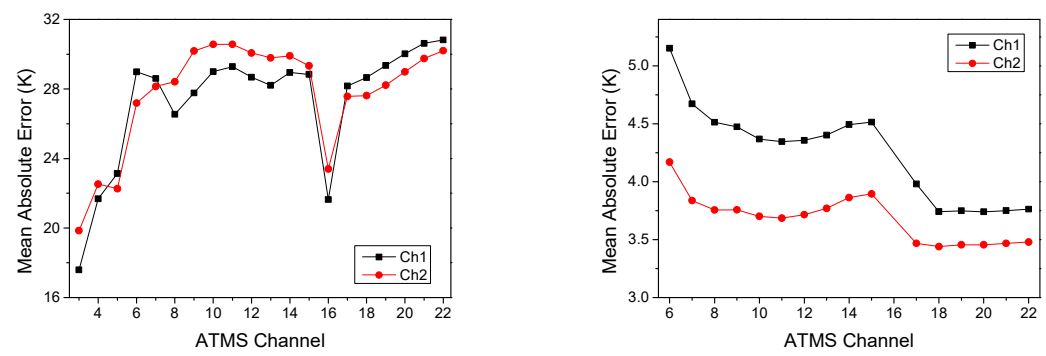

Figure 4. Simulation errors in different channel combinations. (left panel) Mean absolute error of each single channel, (right panel) mean absolute error when each channel is progressively added to the training model. 
https://doi.org/10.5194/amt-2019-447

Preprint. Discussion started: 2 December 2019

(C) Author(s) 2019. CC BY 4.0 License.

(c) (i)
Atmospheric Measurement

Techniques

Discussions
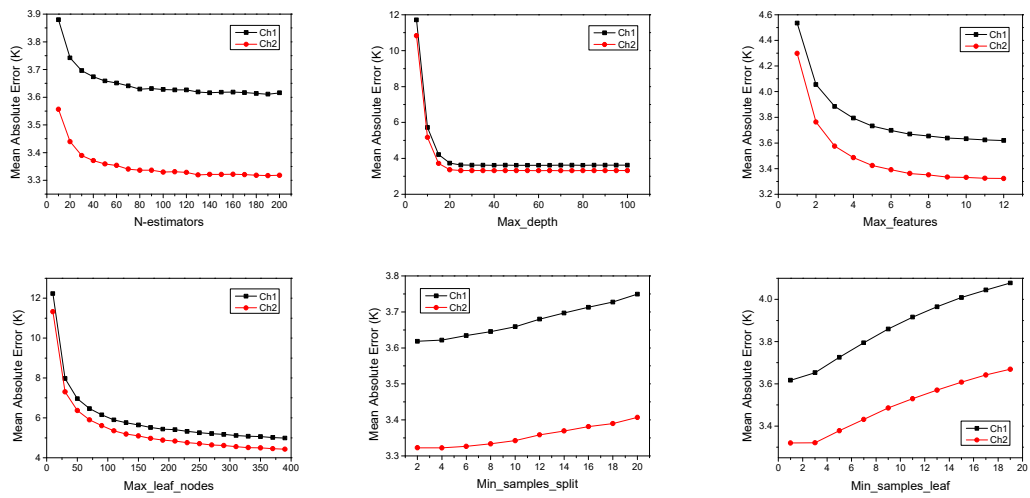

Figure 5. Simulation errors under different RF model parameters. 
https://doi.org/10.5194/amt-2019-447

Preprint. Discussion started: 2 December 2019

(c) Author(s) 2019. CC BY 4.0 License.
Atmospheric Measurement Techniques

Discussions
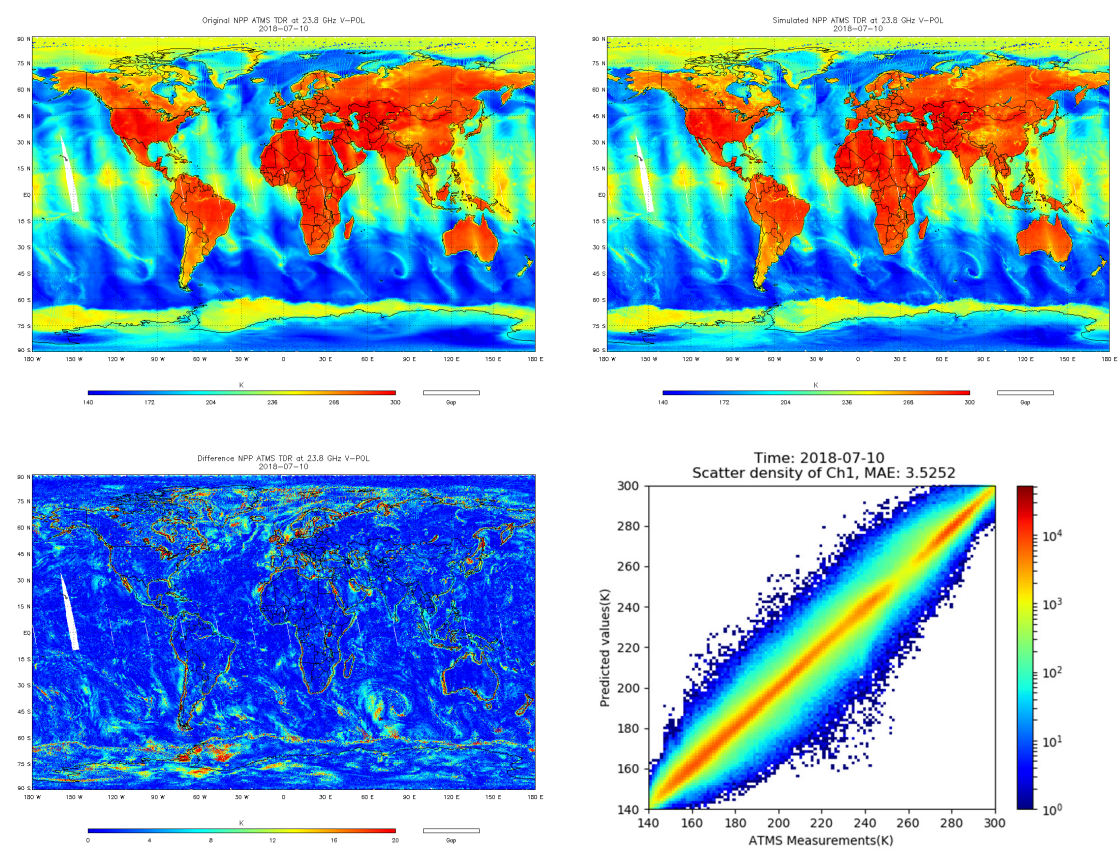

Figure 6. Accuracy evaluation for Ch1 simulation. (left top) Original measurement, (right top) simulated result, (left bottom) difference result, (right bottom) scatter density. 
https://doi.org/10.5194/amt-2019-447

Preprint. Discussion started: 2 December 2019

(C) Author(s) 2019. CC BY 4.0 License.
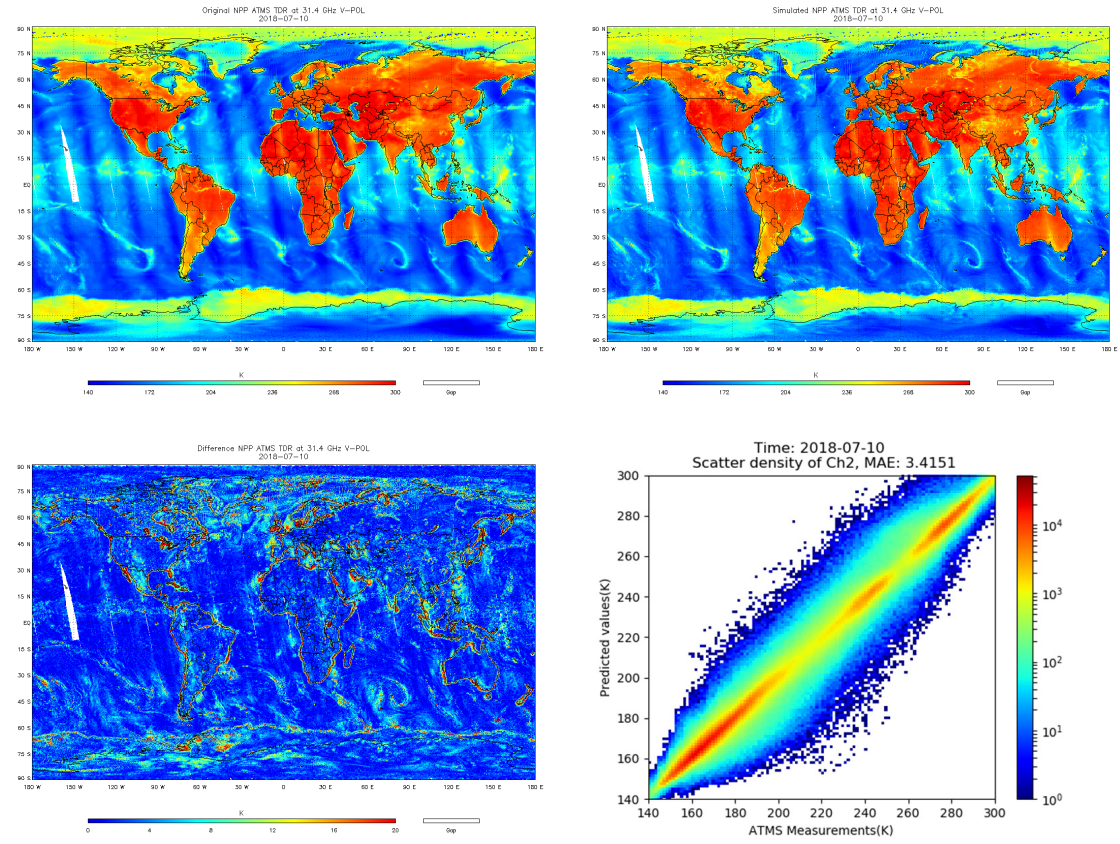

Figure 7. Accuracy evaluation for $\mathrm{Ch} 2$ simulation. (left top) Original measurement, (right top) simulated result, (left bottom) difference result, (right bottom) scatter density. 
https://doi.org/10.5194/amt-2019-447

Preprint. Discussion started: 2 December 2019

(C) Author(s) 2019. CC BY 4.0 License.
Atmospheric Measurement Techniques

Discussions
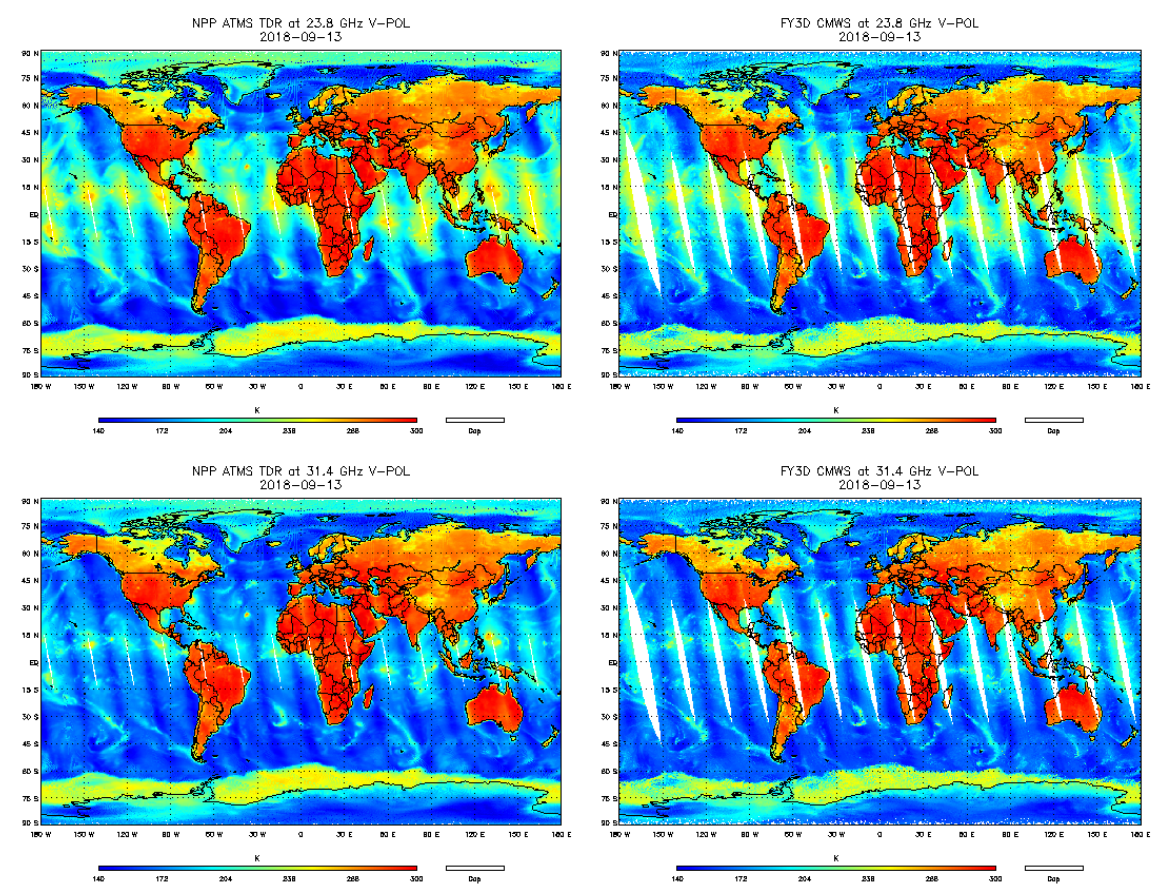

Figure 8. Compared between the simulation and observation. (left column) the original measurements for Ch1 and Ch2, (right column) Simulation results of two corresponding channels based on FY-3D observation. 
https://doi.org/10.5194/amt-2019-447

Preprint. Discussion started: 2 December 2019

(c) Author(s) 2019. CC BY 4.0 License.
Atmospheric Measurement Techniques

Discussions
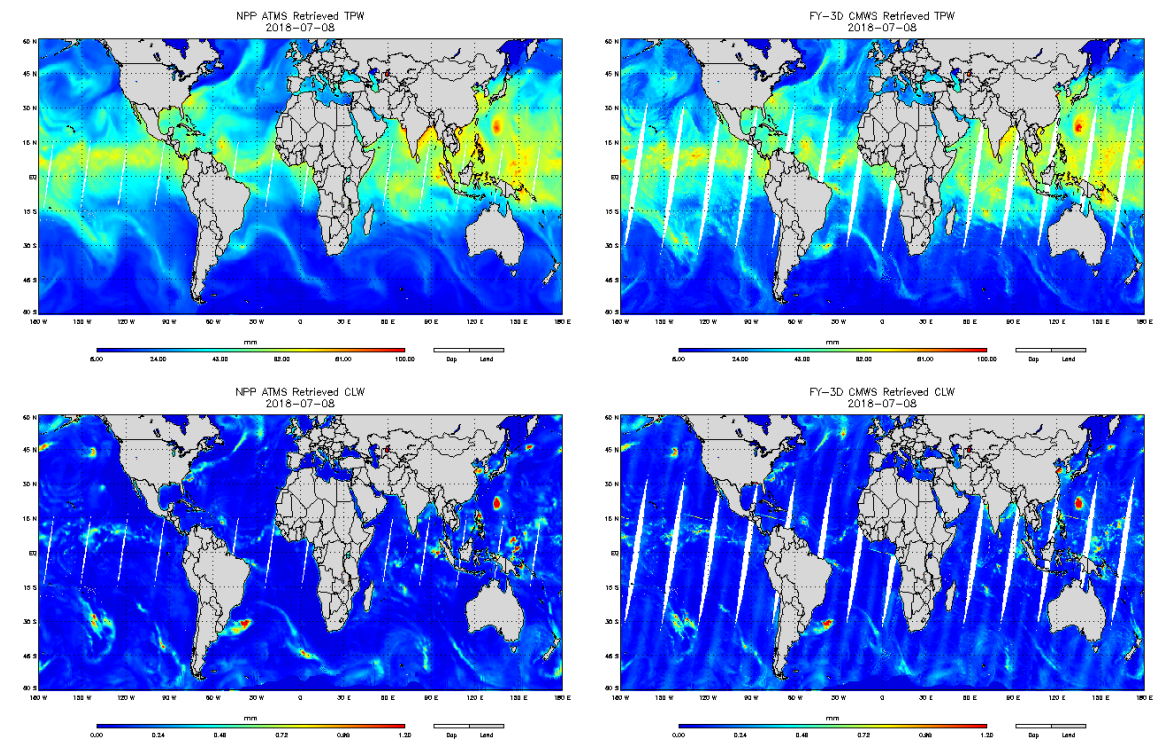

Figure 9. Comparison of retrieved TPW (top row) and CLW (bottom row) between ATMS (left column) and CMWS (right column) for the descending orbit measurement on July 8, 2018. 
https://doi.org/10.5194/amt-2019-447

Preprint. Discussion started: 2 December 2019

(c) Author(s) 2019. CC BY 4.0 License.
Atmospheric Measurement Techniques

Discussions
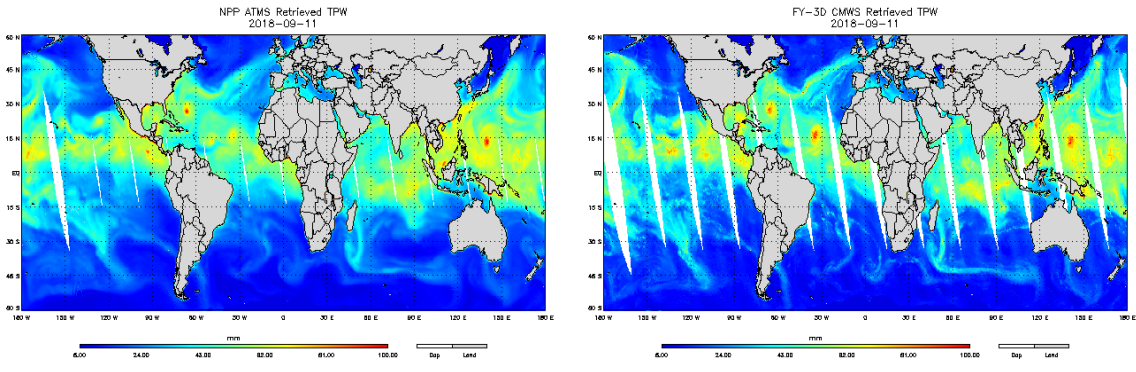

NPP ATMS Retriesed $C L W$
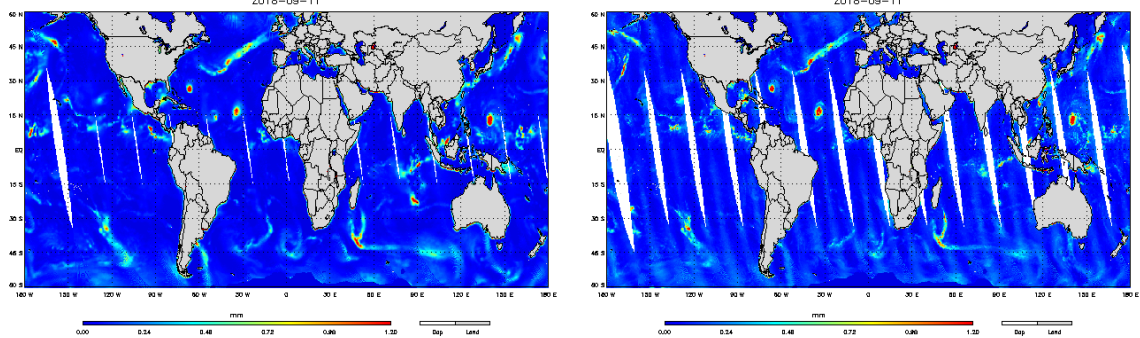

430

Figure 10. Comparison of retrieved TPW (top row) and CLW (bottom row) between ATMS (left column) and CMWS (right column) for the ascending orbit measurement on September 11, 2018. 
https://doi.org/10.5194/amt-2019-447

Atmospheric

Preprint. Discussion started: 2 December 2019

(C) Author(s) 2019. CC BY 4.0 License.

Table 1. Slope, intercept and mean absolute error between corrected CMWS (as $x$ values) and ATMS (as y values)

brightness temperatures.

\begin{tabular}{cccc}
\hline $\begin{array}{c}\text { ATMS Channel } \\
\text { Number }\end{array}$ & Slope & Intercept & $\begin{array}{c}\text { Mean Absolute } \\
\text { Error (K) }\end{array}$ \\
\hline 3 & 1.0124 & -4.6015 & 2.7139 \\
4 & 0.9665 & 8.8694 & 1.4912 \\
5 & 0.9980 & 1.9720 & 1.7411 \\
6 & 1.0021 & 0.0328 & 0.9459 \\
7 & 0.9944 & 1.7225 & 0.9955 \\
8 & 0.9533 & 10.8517 & 0.7353 \\
9 & 0.9978 & -0.7035 & 1.8547 \\
10 & 1.0258 & -6.7331 & 1.8826 \\
11 & 1.0201 & -5.2703 & 1.6061 \\
12 & 1.0163 & -4.2106 & 1.2949 \\
13 & 1.0277 & -8.5121 & 2.6138 \\
14 & 0.9827 & 4.3781 & 1.4696 \\
15 & 1.0255 & -6.6283 & 2.2318 \\
16 & 0.9374 & 18.6856 & 5.0148 \\
17 & 0.9392 & 22.8063 & 8.2390 \\
18 & 0.9747 & 8.0082 & 1.9217 \\
19 & 1.0314 & -10.4440 & 2.9500 \\
20 & 0.9592 & 12.2276 & 1.9781 \\
21 & 0.9311 & 19.6531 & 2.5483 \\
22 & 0.9588 & 13.2642 & 3.2390 \\
\hline
\end{tabular}

